\title{
Molecular detection of Campylobacter species from human and cattle faecal samples in Kilosa district, Tanzania
}

Noel Gahamanyi ( $\nabla$ gahamanyi.noel@sacids.org )

Sokoine University of Agriculture https://orcid.org/0000-0001-7813-6440

Leonard E.G. Mboera

Sokoine University of Agriculture

Mecky I. Matee

Muhimbili University of Health and Allied Sciences

Dieudonné Mutangana

University of Rwanda College of Science and Technology

Raghavendra G. Amachawadi

Kansas City University

Kye-Yoon Yoon

Korea Institute of Science and Technology

Humphrey A. Mabwi

Sokoine University of Agriculture

Kwang Hyun Cha

Korea Institute of Science and Technology

Cheol-Ho Pan

Korea Institute of Science and Technology

Erick V.G. Komba

Sokoine University of Agriculture

\section{Research}

Keywords: Campylobacter, molecular diagnostics, polymerase chain reaction, sequencing, gastroenteritis, Tanzania

Posted Date: July 27th, 2020

DOI: https://doi.org/10.21203/rs.3.rs-46746/v1

License: (c) (i) This work is licensed under a Creative Commons Attribution 4.0 International License.

Read Full License 
Version of Record: A version of this preprint was published at East African Journal of Science, Technology and Innovation on December 17th, 2021. See the published version at https://doi.org/10.37425/eajsti.v3i1.399. 
Molecular detection of Campylobacter species from human and cattle faecal samples in Kilosa district, Tanzania

Noel Gahamanyi ${ }^{1,2^{*}}$, Leonard E.G. Mboera ${ }^{1}$, Mecky I. Matee ${ }^{3}$, Dieudonné Mutangana ${ }^{4}$, Raghavendra G. Amachawadi ${ }^{5}$, Kye-Yoon Yoon ${ }^{2}$, Humphrey A. Mabwi ${ }^{1,2}$, Kwang Hyun Cha ${ }^{2}$, Cheol-Ho Pan ${ }^{2,6}$, Erick V.G. Komba ${ }^{1}$

\section{Abstract}

Background: A growing number of Campylobacter species other than C. jejuni and C. coli have been considered as emerging human and animal pathogens. However, the contribution of these species to human gastroenteritis is poorly documented. This study aimed at detecting Campylobacter species from human and cattle faecal samples in Kilosa district, Tanzania using Polymerase Chain Reaction (PCR) amplification of the 16S rRNA gene, and Sanger sequencing. Methods: A total number of 100 faecal samples ( 70 from human and 30 from cattle) were collected from diarrheic and non-diarrheic patients and healthy cattle in Kilosa district, Tanzania from July to October 2019. Species identification was conducted by PCR and $16 \mathrm{~S}$ rRNA sequencing. The phylogenetic analysis was carried out by comparison of the $16 \mathrm{~S}$ rRNA gene sequences to reference strains by the Neighbor-Joining method in MEGA X.

Results: Campylobacter species detection rate by PCR was 65.7\% (46/70) and 20\% (6/30) in humans and cattle, respectively. There were five human diarrheic cases, four showed Campylobacter presence and two were from children $\leq 15$ years of age. In humans, the $16 \mathrm{~S}$ rRNA sequencing revealed that $C$. concisus was the most predominant species occurring at a frequency of $37.8 \%$ (14/37), followed by uncultured Campylobacter spp. $24.3 \%$ (9/37) and C. hominis $21.6 \%$ (8/37). The least represented species were $C$. jejuni and $C$. lanienae all occurring at 2.7\% (1/37). In cattle, five $(100 \%)$ sequenced PCR products matched with $C$. lanienae. Phylogenetic analysis revealed that Campylobacter $16 \mathrm{~S}$ rRNA sequences were closely related to $C$. concisus, uncultured Campylobacter sp., C. hominis, and C. gracilis.

Conclusion: The non-C. jejuni/ C. coli species are present in human and cattle faecal samples and their true occurrence is probably under-reported due to shortcomings of conventional techniques used in most diagnostic microbiology laboratories. Based on our findings, we recommend that molecular techniques be adopted for direct detection of Campylobacter species during routine laboratory screening and surveillance studies.

Keywords: Campylobacter, molecular diagnostics, polymerase chain reaction, sequencing, gastroenteritis, Tanzania

* Correspondence: gahamanyi.noel@ sacids.org

${ }^{1}$ SACIDS Foundation for One Health, College of Veterinary Medicine and Biomedical Sciences, Sokoine University of Agriculture, Morogoro, Tanzania.

${ }^{2}$ Natural Product Informatics Research Center, KIST Gangneung Institute of Natural Products, Gangneung, Republic of Korea. 


\section{Introduction}

Campylobacter, one of the zoonotic pathogens causing gastroenteritis, is responsible for 96 million cases of diarrhoea each year [1,2]. Campylobacter has also been reported to cause infertility in cattle and abortions in sheep, goats, and cattle [3]. The incidence of human cases of campylobacteriosis has been increasing in many countries throughout the world $[4,5]$. In Africa, the prevalence varies from 7.7-18.5\%, and Campylobacter is persistently found in stools of both diarrheic and non-diarrheic children [7, 8, 9], often associated with poor hygiene and sanitation [9].

Over time, human gastroenteritis has been linked to $C$. jejuni and C. coli accounting for more than 95\% of Campylobacter isolations [11, 12]. However, atypical Campylobacter species are gaining considerable attention as important human and animal pathogens [13-15]. Infections caused by Campylobacter are usually under-reported due to difficulties in isolation procedures [16, 17]. For instance, it has been estimated that $40 \%$ of the bacteria from human faeces diagnosed through microscopy cannot be cultured in the laboratory [17]. In contrast to other gastrointestinal pathogens, the culturing of Campylobacter species is laborious due to their microaerophilic nature and vulnerability to temperature fluctuations [18]. Furthermore, commonly used selective media and added antimicrobials may inhibit the growth of certain Campylobacter species [19]. Moreover, Campylobacter species may become dormant as viable but non-culturable (VBNC) forms difficult to grow on commonly used media [20]. Consequently, the epidemiology and role of non-C. jejuni/ C. coli species to human gastroenteritis are not fully understood $[11,22]$.

The emerging Campylobacter species have been neglected but the integration of molecular techniques and suitable culture media in current diagnostic tests has helped in promoting the awareness of atypical species as relevant human and animal pathogens [5, 22]. Campylobacter concisus has been associated with gastroenteritis, brain abscess, Crohn's disease, arthritis, and ulcerative colitis [14, 23-24]. Campylobacter hominis has been isolated in a blood sample of a septicemic patient [24], while $C$. gracilis has been associated with bacteremia, head infections, periodontitis, and empyema $[23,26]$. In cattle, the most commonly reported species are $C$. fetus, C. lanienae, C. sputorum, C. jejuni, and C. hyointestinalis [28-30].

The polymerase chain reaction (PCR) and other molecular diagnostic tests based on nucleic acids are attractive due to their benefits including their higher sensitivity, ease-of-use, improved turnaround time, relatively low cost, and potential to be fully automated [32-34]. The breakthrough in technology and easy access to commercial kits has led to shifting from traditional laboratory diagnostic techniques to newer molecular ones [32]. The analysis of the 16s rRNA gene by PCR and sequencing techniques has assisted in the phylogenetic identification of Campylobacter species including those unidentified by conventional techniques [27, 35]. Nevertheless, Campylobacter isolation by culture is still useful as it allows the isolation of pure colonies and testing of antimicrobial susceptibilities [34].

In Tanzania and most of the low and middle-income countries (LMICs), the reports on the role of Campylobacter spp. in gastroenteritis are scanty due to limited capacity in laboratory diagnosis and absence of national surveillance programs [7,37]. Thus, the information available for both human and animal campylobacteriosis is limited [36] which undermines its importance as a public health concern. The current study aimed at molecular detection of Campylobacter species from 
human and cattle faecal samples in Kilosa district, Tanzania using PCR amplification of the 16S rRNA gene and Sanger sequencing.

\section{Materials and methods}

\section{Study design and sample collection}

This cross-sectional study was conducted in Kilosa District of central Tanzania $\left(6^{\circ} \mathrm{S}\right.$ and $8^{\circ} \mathrm{S}$, and between $36^{\circ} 30^{\prime}$ and $38^{\circ}$ E) from July 2019 to October 2019. Human stool samples were randomly obtained from patients with abdominal discomfort seeking medical care at Kilosa District Hospital during the time of the study. Cattle faecal samples were randomly collected from healthy lactating cows using sterile gloves. A total of 70 human stool samples and 30 cattle rectal grab faecal samples were collected in sterile dry screw-top containers containing Dimethyl Sulfoxide (DMSO), packed in a cool box and transported to the Sokoine University of Agriculture Microbiology laboratory for DNA extraction within 8 hours of collection.

\section{DNA extraction and Campylobacter species identification}

Approximately, $1 \mathrm{~g}$ faecal sample in DMSO was diluted $(10 \% \mathrm{wt} / \mathrm{vol})$ in buffered peptone water (BPW) $(9 \mathrm{ml})$ and vortexed until the sample was thoroughly homogenized. Then, $200 \mu \mathrm{L}$ of the homogenized faecal sample was used for genomic DNA extraction using Quick-DNA ${ }^{\mathrm{TM}}$ Faecal/Soil Microbe Microprep Kit (Zymo Research Corp, Irvine, CA, USA) based on the manufacturer's instructions. Eluted DNA concentration and purity were checked using a NanoDrop ${ }^{\mathrm{TM}}$ spectrophotometer (Biochrom, Cambridge, England) before storage at $-20^{\circ} \mathrm{C}$.

Detection of Campylobacter was done by multiplex PCR, using C. jejuni cj0414 gene primers (C1; C3) and C. coli ask gene primers (CC18F; CC519R) as previously described [37]. Then, PCR of the 16S rRNA gene was performed on DNA samples negative for $C$. jejuni and $C$. coli using genus-specific primers including a 19bp-forward primer $(\mathrm{C} 412 \mathrm{~F})$ and an 18bp-reverse primer with complementary sequence (C1228R) as previously described [38]. Positive control DNA was extracted from Campylobacter jejuni $\left(\mathrm{ATCC}^{\circledR} 33560^{\mathrm{TM}}\right.$ ); while deionized water was included as the negative control. The PCR final volume was $25 \mu \mathrm{L}$, including $12.5 \mu \mathrm{L}$ of $2 \mathrm{X}$ Master Mix (Thermo Fisher Scientific, Seoul, South Korea), $1 \mu \mathrm{L}(10 \mu \mathrm{M})$ of C412F primer, $1 \mu \mathrm{L}(10 \mu \mathrm{M})$ of C1228R primer, $1 \mu \mathrm{L}$ of template DNA, and $9.5 \mu \mathrm{L}$ of sterile deionized water. All primers were made by Integrated DNA Technologies, Inc. (Singapore Science Park, Singapore).

The DNA amplification was performed using the model MiniAmp ${ }^{\mathrm{TM}}$ Plus Thermal Cycler (Applied Biosystems, MA, USA). The cycling conditions used were one cycle of $95^{\circ} \mathrm{C}$ for 5 minutes, 35 cycles each of $94^{\circ} \mathrm{C}$ for 30 seconds, $55^{\circ} \mathrm{C}$ for 45 seconds and $72^{\circ} \mathrm{C}$ for 45 seconds, and a final extension at $72^{\circ} \mathrm{C}$ for 7 minutes. The PCR products were held at $4^{\circ} \mathrm{C}$ before analysis. PCR products $(5 \mu l)$ were mixed with $2 \mu$ l Loading STAR (Dyne bio, Seongnam-si, Korea) diluted with $5 \mu 1$ of nuclease-free water and analyzed by gel electrophoresis: $10 \mu 1$ of the mixture was loaded onto $1.5 \%$ SeaKem ${ }^{\circledR}$ LE Agarose gel (Lonza Inc.-Rockland, ME, USA) in 0.5X TAE buffer. After electrophoresis, PCR product bands were visualized using a Dual UV Transilluminator (Core Bio System, Huntington Beach, CA, USA) under ultraviolet (UV) light and photographed with iBright ${ }^{\text {TM }}$ CL1000 Imaging System (Thermo Fisher Scientific, Seoul, South Korea). The size of the amplification products (816bp) obtained was compared to the Dyne $100 \mathrm{bp}$ DNA ladder (Dyne bio, Seongnam-si, Korea). The PCR products (816 bp) were purified using Pure Link ${ }^{\mathrm{TM}}$ Quick 
PCR purification Kit (Invitrogen, Vilnius, Lithuania) and sequenced at SolGent (Solutions for Genetic technologies, Daejeon, South Korea) using the Campylobacter genus-specific primers by Sanger method.

\section{Data analysis}

The data were analyzed with GraphPad Prism 8.4.0 (GraphPad Software, La Jolla, CA, USA). Descriptive statistics (frequencies and percentage) were computed to determine proportions for different attributes. The GenBank sequences with the best, and the high scoring matches with sequences of this study were selected using the NCBI BLASTN search. Sequences were edited, aligned, and analyzed using BioEdit sequence alignment software (version 7.2.6.1) [40, 41]. Multiple sequence alignment by Muscle [41], computation of evolutionary distances by the JukesCantor method [42], and the phylogenetic tree building by the Neighbor-Joining (NJ) method [43], were done in MEGA X software (MEGA Inc, Englewood, NJ) [44]. The phylogenetic analysis was carried out by comparing the sequences of this study to 16S rRNA genes of reference strains downloaded from LPSN (https://lpsn.dsmz.de). To confirm the reliability of our analysis, bootstrap analysis was performed with 1,000 resampled datasets and it was taken to represent the evolutionary history of the taxa analyzed [45]. All the 16S rRNA gene sequences derived from sequencing were submitted to GenBank for obtaining accession numbers.

\section{Results}

A total of 70 (male $=35$; female $=35$ ) human stool samples were collected. The age of patients ranged from 2 to 89 years with $14.3 \%$ being children $\leq 15$ years of age. Overall, the detection rate of Campylobacter spp. in human samples was $65.7 \%$. The PCR products with predicted size (816bp) were obtained in some of the screened samples (Figure 1). Of the Campylobacter spp. positive samples $(\mathrm{n}=46), 24(52.2 \%)$ were from females and $22(47.8 \%)$ were from males. Campylobacter species were detected in nine of the $10(90 \%)$ children $\leq 15$ years old. There were five diarrheic cases, of which four showed Campylobacter presence. Of the diarrheic patients, two were children $\leq 15$ years. In cattle, all the 30 samples were collected from lactating cows with age varying between 3.4-8 years. Of the 30 samples, six (20\%) exhibited Campylobacter spp.

Figure 1: PCR products using the 16S rRNA primers specific for Campylobacter genus. Lanes: 1: 100bp molecular weight marker; 2-4: bands from human samples; 5: negative control; 6: positive control; 7-8: bands from cattle samples (Attachment).

The results of sequencing confirmed the presence of Campylobacter species in all submitted sequences (37 from humans and 5 from cattle). The remaining PCR products did not give enough quantity of DNA (required by the sequencing company) after the purification step. The species were confirmed based on percent identity (above 99\%), the query cover, and the E-value. In humans, C. concisus was the most prevalent (37.8\%), followed by uncultured Campylobacter spp. (24.3\%), and C. hominis (21.6\%). Campylobacter lanienae, and C. jejuni occurred at a frequency of $2.7 \%$ each (Figure 2). For cattle, all the five (100\%) 16S rRNA sequences matched with C. lanienae. 
Figure 2: Distribution of Campylobacter species from human samples identified by sequencing (Attachment).

The 16S rRNA genes of Campylobacter spp. from this study were compared with 16S rRNA sequences of different strains of Campylobacter spp. by BLASTN search. Following submission to the GenBank, gene sequences were allocated with the following accession numbers: MT126449 to MT126453; MT130973 to MT130991; and MT131150 to MT131167.

The phylogenetic analysis was carried out by comparing the 16S rRNA genes of this study to 16S rRNA genes of reference strains (C. concisus, C. hominis, C. gracilis, and C. lanienae) and uncultured Campylobacter. The analysis of sequence data from Campylobacter species of this study revealed a high nucleotide sequence similarity to different reference strains. Campylobacter hominis clustered closer to $C$. gracilis than it was with $C$. concisus. It was also noted that C. lanienae formed a separate cluster at the bottom of the tree. Uncultured Campylobacter and Campylobacter spp. RM 12175 were also found among the sequences of this study (Figure 3). The tree was rooted using $H$. aurati and A. molluscorum.

Figure 3: Molecular phylogenetic analysis of Campylobacter species using 16S rRNA sequences by the neighbor-joining method. Bootstrap values (\%) based on 1000 replicates are indicated at nodes. The evolutionary distances were computed using the Jukes-Cantor method and are in the units of the number of base substitutions per site. Evolutionary analyses were conducted in MEGA X. Reference strains were included in the analysis. The tree was rooted using $H$. aurati and A. molluscorum (Attachment).

\section{Discussion}

For many years $C$. jejuni and $C$. coli have been the most commonly reported species associated with gastroenteritis in humans [11]. Nonetheless, advances in molecular diagnostic techniques proved that isolation by culture contributes to increased detection rates of $C$. jejuni and $C$. coli over other species which bias both the outcome of the diagnosis and the contribution of other species to Campylobacter infections [46]. The routine culture-based method fails to detect over a third of Campylobacter positive samples. Bullman et al. [46] attribute the scarcity of atypical Campylobacter species reported so far to the limitations and bias of culture-based methods. Therefore, this study reports the detection of Campylobacter spp. in both humans and cattle by molecular methods. To the best of our knowledge, this is the first report in Tanzania detecting the presence of Campylobacter species directly from feces without a culture-based approach. Previous authors adopted culture methods [49-51] which could not give a true picture of Campylobacterrelated infections as some could have failed to grow on selective media.

The reported detection rate of Campylobacter spp. (65.7\%) in humans was similar to that reported in Nigeria [50] but higher than those reported previously in Tanzania [53, 54], Fiji [53], India [54], and Cambodia [9]. The difference could be attributed to the sampling strategy, PCR conditions, and geographically related variations. Campylobacter concisus and $C$. hominis were the most predominant species occurring at $37.8 \%$ and $21.6 \%$, respectively. This concurs with the findings reported in Denmark [55] and Australia [56]. Lastovica et al. [16] reported that $C$. concisus was the second species with a higher prevalence after $C$. jejuni. However, $C$. concisus has been isolated 
from diarrheic patients without other pathogenic microorganisms suggesting it to be an emerging cause of human gastroenteritis [16,57].

Campylobacter lanienae was detected in both cattle and humans. It has been previously recovered from healthy livestock [57]. This Campylobacter spp. has been reported as a probable aetiology of human gastroenteritis [58]. However, it is suggested that $C$. lanienae has restricted pathogenicity or be a non-pathogenic Campylobacter [22]. The observed frequency of occurrence of the species in this study was higher than the one previously reported by Inglis \& Kalischuk [28]. Further characterization of $C$. lanienae could shed more light on its genetic diversity and source [57].

The data on the concurrent isolation of Campylobacter species in both humans and cattle are limited. In this study, the detection rates were $65.7 \%$ and $20 \%$, in humans and cattle, respectively. Our findings showed higher detection rates when compared to the rates reported earlier in Tanzania [59], and Cambodia [9]. Further comparative studies on Campylobacter species isolated from humans and cattle are necessary to understand their epidemiology and be able to conclude on source attribution.

The phylogenetic analysis based on the 16S rRNA gene is of paramount importance for bacterial taxonomy [60] and it has been applied to Brucella [61] and Campylobacter identification [5, 61]. Our findings concur with the reported species of non-C. jejunil $C$. coli group with $C$. concisus being the predominant species [63]. In cattle, our results are in agreement with previously reported occurrence where C. lanienae had higher proportions compared to C. jejuni and C. hyointestinalis [57].

The current study had some limitations including the sample size and lack of culture-based species identification. The sample size used could not allow us to estimate the prevalence or generalize the findings at national or regional levels. Considering that we did not culture the stool samples, the comparison is made based on previous studies carried in sometimes different conditions or settings. However, this study highlights the advantages of molecular methods over the culturebased one.

\section{Conclusions}

The findings of this study highlight the higher prevalence of less frequently isolated Campylobacter species (C. concisus and C. hominis) in patients with gastroenteritis. Campylobacter lanienae was detected in both human and cattle faecal samples. These Campylobacter species are often neglected due to their cultural behavior and fastidious nature but have proven to be zoonotic with a public health concern. It is therefore important that health practitioners and public health authorities recognize the possibilities of occurrence of Campylobacter species other than C. jejuni and C. coli which are not tested on a routine basis in many countries and go unreported. Molecular-based techniques offer an alternative to culturebased methods especially when it comes to the atypical Campylobacter species as they provide results in a short time and up to species level. 


\section{List of abbreviations}

PCR: polymerase chain reaction; NJ: Neighbor-Joining method; MEGA: Molecular Evolutionary Genetics Analysis; LMICs: low and middle-income countries; 16S rRNA: 16S ribosomal ribonucleic acid, where $\mathrm{S}$ stands for Svedberg.

\section{Declarations}

\section{Ethics approval and consent to participate}

This study was approved by the Medical Research Coordinating Committee of the National Institute for Medical Research in Tanzania (Ref. No.: NIMR/HQ/R.8a/Vol. IX/3070). Stool samples from patients were collected after obtaining informed written consent of the patient or his/her parent/guardian. Farmers consented for sampling their cattle.

\section{Consent for publication \\ Not applicable}

\section{Availability of data and materials}

Data generated during this study are available from the corresponding author on reasonable request

\section{Competing interests}

The authors declare that they have no competing interests.

\section{Funding \\ Not applicable}

\section{Authors' contributions}

NG carried out the experiments, compiled the results, and wrote the manuscript. KYY and HAM participated in carrying out the experiments. EVGK, MIM, LEGM, RGA, DM, CHP, and KHC participated in the design and helped to revise the manuscript. All authors approved the final version of the manuscript.

\section{Acknowledgments}

We gratefully acknowledge the Partnership for Skills in Applied Sciences, Engineering and Technology, the SACIDS Foundation for One Health, and the Korea Institute for Science and Technology for their contribution towards this study and its publication.

\section{Author details}

${ }^{1}$ SACIDS Foundation for One Health, College of Veterinary Medicine and Biomedical Sciences, Sokoine University of Agriculture, Chuo Kikuu, Morogoro, Tanzania. ${ }^{2}$ Natural Product Informatics Research Center, KIST Gangneung Institute of Natural Products, Gangneung, Republic of Korea. ${ }^{3}$ School of Medicine, Muhimbili University of Health and Allied Sciences, Dar es Salaam, Tanzania. ${ }^{4}$ College of Science and Technology, University of Rwanda, Kigali, Rwanda. ${ }^{5}$ Department of Clinical Sciences, College of Veterinary Medicine, Kansas State University, Manhattan, Kansas, United States. ${ }^{6}$ Division of Bio-Medical Science and Technology, KIST School, Korea University of Science and Technology, Seoul 02792, Republic of Korea. 


\section{References}

[1] G. M. Ruiz-Palacios, "The Health Burden of Campylobacter Infection and the Impact of Antimicrobial Resistance: Playing Chicken," Clin. Infect. Dis., vol. 44, pp. 701-703, 2007, doi: 10.1086/509936.

[2] A. H. Havelaar et al., "World Health Organization Global Estimates and Regional Comparisons of the Burden of Foodborne Disease in 2010," PLOS Med., vol. 12, no. 12, p. e1001923, 2015, doi: 10.1371/journal.pmed.1001923.

[3] O. Sahin, M. Yaeger, Z. Wu, and Q. Zhang, "Campylobacter-associated diseases in animals," Annu. Rev. Anim. Biosci., vol. 5, pp. 21-42, 2017, doi: 10.1146/annurev-animal-022516022826.

[4] N. O. Kaakoush, N. Castaño-Rodríguez, and H. M. Mitchell, "Global epidemiology of Campylobacter infection," Clin. Microbiol. Rev., vol. 28, pp. 687-720, 2015, doi: 10.1128/CMR.00006-15.

[5] I. M. Sulaiman, Y.-H. Hsieh, and S. Simpson, "Species identification of Campylobacter jejuni and Campylobacter coli isolates from raw poultry products by MALDI-TOF MS and rRNA Sequence analysis," J. Aoac Int., vol. 103, no. 1, pp. 197-204, 2020, doi: 10.5740/jaoacint.19-0170.

[6] A. O. Coker, R. D. Isokpehi, B. N. Thomas, K. O. Amisu, and C. L. Obi, "Human campylobacteriosis in developing countries," Emerg. Infect. Dis., vol. 8, pp. 237-243, 2002.

[7] R. V. Randremanana et al., "Campylobacter infection in a cohort of rural children in Moramanga, Madagascar," BMC Infect. Dis., vol. 14, no. 372, pp. 1-9, 2014.

[8] N. Gahamanyi, L. E. G. Mboera, M. I. Matee, D. Mutangana, and E. V. G. Komba, "Prevalence, risk factors, and antimicrobial resistance profiles of thermophilic Campylobacter species in humans and animals in Sub-Saharan Africa: A systematic review," Int. J. Microbiol., vol. 2020, pp. 1-12, 2020, doi: 10.1155/2020/2092478.

[9] K. Osbjer et al., "Detection of Campylobacter in human and animal field samples in Cambodia," APMIS, vol. 124, pp. 508-515, 2016, doi: 10.1111/apm.12531.

[10] S. P. Kulkarni, "Detection of campylobacter species: a comparison of culture and polymerase chain reaction based methods," J. Clin. Pathol., vol. 55, pp. 749-753, 2002, doi: 10.1136/jcp.55.10.749.

[11] J. E. Moore et al., "Campylobacter," Vet Res, vol. 36, pp. 351-382, 2005, doi: 10.1051/vetres:2005012.

[12] A. J. Lastovica, "Emerging Campylobacter spp.: The tip of the iceberg," Clin. Microbiol. Newsl., vol. 28, no. 7, pp. 49-56, 2006, doi: 10.1016/j.clinmicnews.2006.03.004.

[13] L. Zhang et al., "Detection and isolation of Campylobacter species other than C. jejuni from Children with Crohn's Disease," J. Clin. Microbiol., vol. 47, pp. 453-455, 2009, doi: 10.1128/JCM.01949-08.

[14] S. M. Man, "The clinical importance of emerging Campylobacter species," Nat. Rev. Gastroenterol. Hepatol., vol. 8, pp. 669-685, 2011, doi: 10.1038/nrgastro.2011.191.

[15] J. Engberg, S. L. W. On, C. S. Harrington, and P. Gerner-Smidt, "Prevalence of Campylobacter, Arcobacter, Helicobacter, and Sutterella spp. in human fecal samples as estimated by a reevaluation of isolation methods for Campylobacters," J. Clin. Microbiol., vol. 38, pp. 286-291, 2000.

[16] A. J. Lastovica, "Non-jejuni/coli Campylobacter species and related organisms in poultry, and their relevance in human and animal disease," in Campylobacter spp. and Related 
Organisms in Poultry, B. B. Fonseca, H. Fernandez, and D. A. Rossi, Eds. Cham: Springer International Publishing, 2016, pp. 185-206.

[17] R. D. Berg, "The indigenous gastrointestinal microflora," Trends Microbiol., vol. 4, pp. 430435, 1996, doi: 10.1016/0966-842X(96)10057-3.

[18] S. F. Park, "The physiology of Campylobacter species and its relevance to their role as foodborne pathogens," Int. J. Food Microbiol., vol. 74, pp. 177-188, 2002.

[19] E. Bessede, A. Delcamp, E. Sifre, A. Buissonniere, and F. Megraud, "New methods for detection of Campylobacters in stool samples in comparison to culture," J. Clin. Microbiol., vol. 49, pp. 941-944, 2011, doi: 10.1128/JCM.01489-10.

[20] H. Singh, R. S. Rathore, S. Singh, and P. S. Cheema, "Comparative analysis of cultural isolation and PCR based assay for detection of Campylobacter jejuni in food and faecal samples," Braz. J. Microbiol., vol. 42, pp. 181-186, Mar. 2011, doi: 10.1590/S151783822011000100022.

[21] S. Bullman, D. Corcoran, J. O’Leary, D. O'Hare, B. Lucey, and R. D. Sleator, "Emerging dynamics of human campylobacteriosis in Southern Ireland," FEMS Immunol. Med. Microbiol., vol. 63, pp. 248-253, 2011, doi: 10.1111/j.1574-695X.2011.00847.x.

[22] D. Costa and G. Iraola, "Pathogenomics of Emerging Campylobacter Species," Clin. Microbiol. Rev., vol. 32, no. 4, 2019, doi: 10.1128/CMR.00072-18.

[23] F. Liu, R. Ma, Y. Wang, and L. Zhang, "The clinical importance of Campylobacter concisus and other human hosted Campylobacter species," Front. Cell. Infect. Microbiol., vol. 8, pp. 1-22, 2018, doi: 10.3389/fcimb.2018.00243.

[24] A. J. Linscott, R. B. Flamholtz, D. Shukla, Y. Song, C. Liu, and S. M. Finegold, "Fatal septicemia due to Clostridium hathewayi and Campylobacter hominis," Anaerobe, vol. 11, pp. 97-98, 2005, doi: 10.1016/j.anaerobe.2004.10.002.

[25] T. Shinha, "Fatal bacteremia caused by Campylobacter gracilis, United States," Emerg. Infect. Dis., vol. 21, pp. 1084-1085, 2015, doi: 10.3201/eid2106.142043.

[26] G. Gorkiewicz et al., "Species-specific identification of Campylobacters by partial 16S rRNA gene sequencing," J. Clin. Microbiol., vol. 41, pp. 2537-2546, 2003, doi: 10.1128/JCM.41.6.2537-2546.2003.

[27] H. I. Atabay and J. E. Corry, "The isolation and prevalence of campylobacters from dairy cattle using a variety of methods," J. Appl. Microbiol., vol. 84, pp. 733-740, 1998, doi: 10.1046/j.1365-2672.1998.00402.x.

[28] G. D. Inglis and L. D. Kalischuk, "Use of PCR for direct detection of Campylobacter species in bovine feces," Appl. Environ. Microbiol., vol. 69, pp. 3435-3447, 2003, doi: 10.1128/AEM.69.6.3435-3447.2003.

[29] G. D. Mshelia, J. D. Amin, Z. Woldehiwet, R. D. Murray, and G. O. Egwu, "Epidemiology of bovine venereal Campylobacteriosis: Geographic distribution and recent advances in molecular diagnostic techniques," Reprod. Domest. Anim., vol. 45, pp. e221-e230, 2010, doi: 10.1111/j.1439-0531.2009.01546.x.

[30] D. Linton, A. J. Lawson, R. J. Owen, and J. Stanley, "PCR detection, identification to species level, and fingerprinting of Campylobacter jejuni and Campylobacter coli direct from diarrheic samples," J Clin Microbiol, vol. 35, pp. 2568-2572, 1997.

[31] M. J. LaGier, L. A. Joseph, T. V. Passaretti, K. A. Musser, and N. M. Cirino, "A real-time multiplexed PCR assay for rapid detection and differentiation of Campylobacter jejuni and Campylobacter coli," Mol. Cell. Probes, vol. 18, pp. 275-282, 2004, doi: 10.1016/j.mcp.2004.04.002. 
[32] M. Amjad, "An overview of the molecular methods in the diagnosis of gastrointestinal infectious diseases," Int. J. Microbiol., vol. 2020, pp. 1-13, 2020, doi: $10.1155 / 2020 / 8135724$.

[33] B. E. Anderson, J. E. Dawson, D. C. Jones, and K. H. Wilson, "Ehrlichia chaffeensis, a new species associated with human ehrlichiosis.," J. Clin. Microbiol., vol. 29, pp. 2838-2842, 1991.

[34] R. Ghosh, B. Uppal, P. Aggarwal, A. Chakravarti, A. K. Jha, and A. P. Dubey, "A Comparative study of conventional and molecular techniques in diagnosis of Campylobacter gastroenteritis in children," Ann. Clin. Lab. Sci., vol. 44, no. 1, pp. 42-48, 2014.

[35] P. Padungton and J. B. Kaneene, "Campylobacter spp. in human, chickens, pigs and their antimicrobial resistance," J. Vet. Med. Sci., vol. 65, pp. 161-170, 2003.

[36] E. V. G. Komba, R. H. Mdegela, P. L. Msoffe, and H. Ingmer, "Human and animal Campylobacteriosis in Tanzania: A review," Tanzan. J. Health Res., vol. 15, no. 1, pp. 1-13, 2013, doi: 10.4314/thrb.v15i1.6.

[37] W. Yamazaki-Matsune et al., "Development of a multiplex PCR assay for identification of Campylobacter coli, Campylobacter fetus, Campylobacter hyointestinalis subsp. hyointestinalis, Campylobacter jejuni, Campylobacter lari and Campylobacter upsaliensis," J. Med. Microbiol., vol. 56, no. 11, pp. 1467-1473, 2007.

[38] D. Linton, R. J. Owen, and J. Stanley, "Rapid identification by PCR of the genus Campylobacter and of five Campylobacter species enteropathogenic for man and animals," Res. Microbiol., vol. 147, pp. 707-718, 1996, doi: 10.1016/S0923-2508(97)85118-2.

[39] T. A. Hall, "Bioedit: a user-friendly biological sequence alignment editor and analysis program for windows 95/98/NT," Nucl Acids Symp Serm, vol. 41, pp. 95-98, 1999.

[40] T. Madden, "The BLAST sequence analysis tool.," in The NCBI Handbook [Internet]. 2nd edition, National Center for Biotechnology Information (US)., 2013, pp. 1-10.

[41] R. C. Edgar, "MUSCLE: multiple sequence alignment with high accuracy and high throughput," Nucleic Acids Res., vol. 32, pp. 1792-1797, 2004, doi: 10.1093/nar/gkh340.

[42] T. H. Jukes and C. R. Cantor, "Evolution of protein molecules," in Mammalian protein metabolism, 21st ed., 3 vols., H. N. Munro, Ed. Academic Press, New York, 1969, pp. 21132.

[43] N. Saitou and M. Nei, "The neighbor-joining method: a new method for reconstructing phylogenetic trees.," Mol. Biol. Evol., vol. 4, pp. 406-425, 1987, doi: 10.1093/oxfordjournals.molbev.a040454.

[44] S. Kumar, G. Stecher, M. Li, C. Knyaz, and K. Tamura, "MEGA X: Molecular Evolutionary Genetics Analysis across computing platforms," Mol. Biol. Evol., vol. 35, pp. 1547-1549, 2018, doi: 10.1093/molbev/msy096.

[45] J. Felsenstein, "Confidence limits on phylogenies: An approach using the bootstrap," Evolution, vol. 39, pp. 783-791, 1985, doi: 10.1111/j.1558-5646.1985.tb00420.x.

[46] S. Bullman, J. O'Leary, D. Corcoran, R. D. Sleator, and B. Lucey, "Molecular-based detection of non-culturable and emerging campylobacteria in patients presenting with gastroenteritis," Epidemiol. Infect., vol. 140, pp. 684-688, 2012, doi: 10.1017/S0950268811000859.

[47] R. H. Mdegela, H. E. Nonga, H. A. Ngowi, and R. R. Kazwala, "Prevalence of thermophilic Campylobacter infections in humans, chickens and crows in Morogoro, Tanzania," J. Vet. Med. Ser. B, vol. 53, pp. 116-121, 2006. 
[48] I. P. Kashoma et al., "Antimicrobial resistance and genotypic diversity of Campylobacter isolated from pigs, dairy, and beef cattle in Tanzania," Front. Microbiol., vol. 6, pp. 1-11, 2015.

[49] E. V. G. Komba, R. H. Mdegela, P. L. M. Msoffe, L. N. Nielsen, and H. Ingmer, "Prevalence, antimicrobial resistance and risk factors for thermophilic Campylobacter infections in symptomatic and asymptomatic humans in Tanzania," Zoonoses Public Health, vol. 62, pp. 557-568, 2015.

[50] P. B. Gwimi et al., "Prevalence of Campylobacter species in fecal samples of pigs and humans from Zuru Kebbi State, Nigeria," Int. J. One Health, vol. 1, pp. 1-5, 2015.

[51] P. Jacob, R. H. Mdegela, and H. E. Nonga, "Comparison of Cape Town and Skirrow's Campylobacter isolation protocols in humans and broilers in Morogoro, Tanzania," Trop. Anim. Health Prod., vol. 43, pp. 1007-1013, Jun. 2011, doi: DOI 10.1007/s11250-011-9799$\mathrm{z}$.

[52] I. S. Chuma, H. E. Nonga, R. H. Mdegela, and Rudovick. R. Kazwala, "Epidemiology and RAPD-PCR typing of thermophilic campylobacters from children under five years and chickens in Morogoro Municipality, Tanzania," BMC Infect. Dis., vol. 16, pp. 1-11, Nov. 2016.

[53] A. Devi, J. Wilkinson, T. Mahony, and T. Vanniasinkam, "Detection of Campylobacter in human faecal samples in Fiji," WPSAR, vol. 5, no. 4, pp. 30-33, 2014, doi: 10.5365/WPSAR.2014.5.2.007.

[54]S. M. Salim, J. Mandal, and S. Parija, "Isolation of Campylobacter from human stool samples," Indian J. Med. Microbiol., vol. 32, no. 1, pp. 35-38, 2014, doi: 10.4103/0255-0857.124294.

[55] R. Aabenhus, H. Permin, S. L. W. On, and L. P. Andersen, "Prevalence of Campylobacter concisus in Diarrhoea of Immunocompromised Patients," Scand. J. Infect. Dis., vol. 34, no. 4, pp. 248-252, 2002, doi: 10.1080/00365540110080566.

[56] V. Mahendran et al., "Prevalence of Campylobacter species in adult Crohn's Disease and the preferential colonization sites of Campylobacter species in the human intestine," PLoS ONE, vol. 6, no. 9, pp. 1-7, 2011, doi: 10.1371/journal.pone.0025417.

[57] E. Guévremont, V. Normand, L. Lamoureux, and C. Côté, "Genetic detection of Campylobacter lanienae in fecal matter and stored manure from swine and dairy cattle," Foodborne Pathog. Dis., vol. 5, pp. 361-364, 2008, doi: 10.1089/fpd.2007.0054.

[58] S. Lévesque, F. Lemay, S. Bekal, E. H. Frost, and S. Michaud, "First reported case of Campylobacter lanienae enteritis in a human," JMM Case Rep., vol. 3, no. 3, pp. 1-5, 2016, doi: 10.1099/jmmcr.0.005045.

[59] L. J. M. Kusiluka et al., "Prevalence and impact of water-borne zoonotic pathogens in water, cattle and humans in selected villages in Dodoma Rural and Bagamoyo districts, Tanzania," Phys. Chem. Earth Parts ABC, vol. 30, no. 11-16, pp. 818-825, 2005.

[60] F. E. Dewhirst et al., "Discordant 16S and 23S rRNA gene phylogenies for the genus Helicobacter: implications for phylogenetic inference and systematics," J. Bacteriol., vol. 187, pp. 6106-6118, 2005, doi: 10.1128/JB.187.17.6106-6118.2005.

[61] J.-B. Ntirandekura et al., "Molecular characterization of Brucella species detected in humans and domestic ruminants of pastoral areas in Kagera ecosystem, Tanzania," Vet. Med. Sci., pp. 1-9, 2020, doi: 10.1002/vms3.298.

[62] A. M. Boukerb et al., "Campylobacter armoricus sp. nov., a novel member of the Campylobacter lari group isolated from surface water and stools from humans with enteric infection," Int. J. Syst. Evol. Microbiol., vol. 69, no. 12, pp. 3969-3979, 2019, doi: 10.1099/ijsem.0.003836. 
513 [63] I. Mukhopadhya, J. M. Thomson, R. Hansen, S. H. Berry, E. M. El-Omar, and G. L. Hold,

514

515

516

517

518
"Detection of Campylobacter concisus and other Campylobacter Species in colonic biopsies from adults with Ulcerative Colitis," PLOS ONE, vol. 6, no. 6, pp. 1-7, 2011, doi: 10.1371/journal.pone.0021490. 
Figures

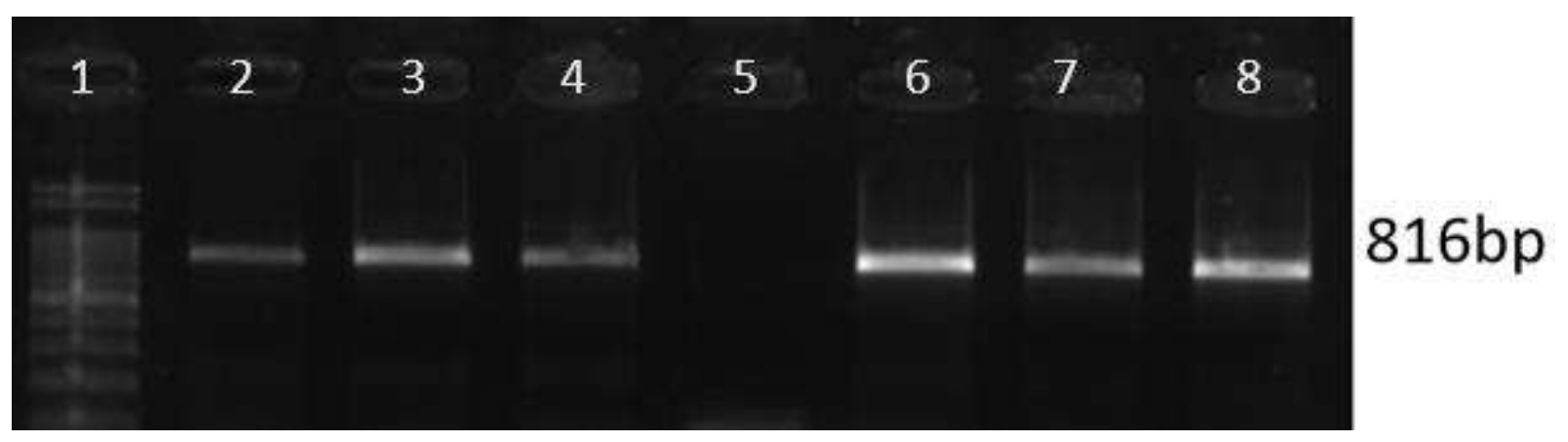

\section{Figure 1}

PCR products using the 16S rRNA primers specific for Campylobacter genus. Lanes: 1: 100bp molecular weight marker; 2-4: bands from human samples; 5 : negative control; 6: positive control; 7-8: bands from cattle samples. 


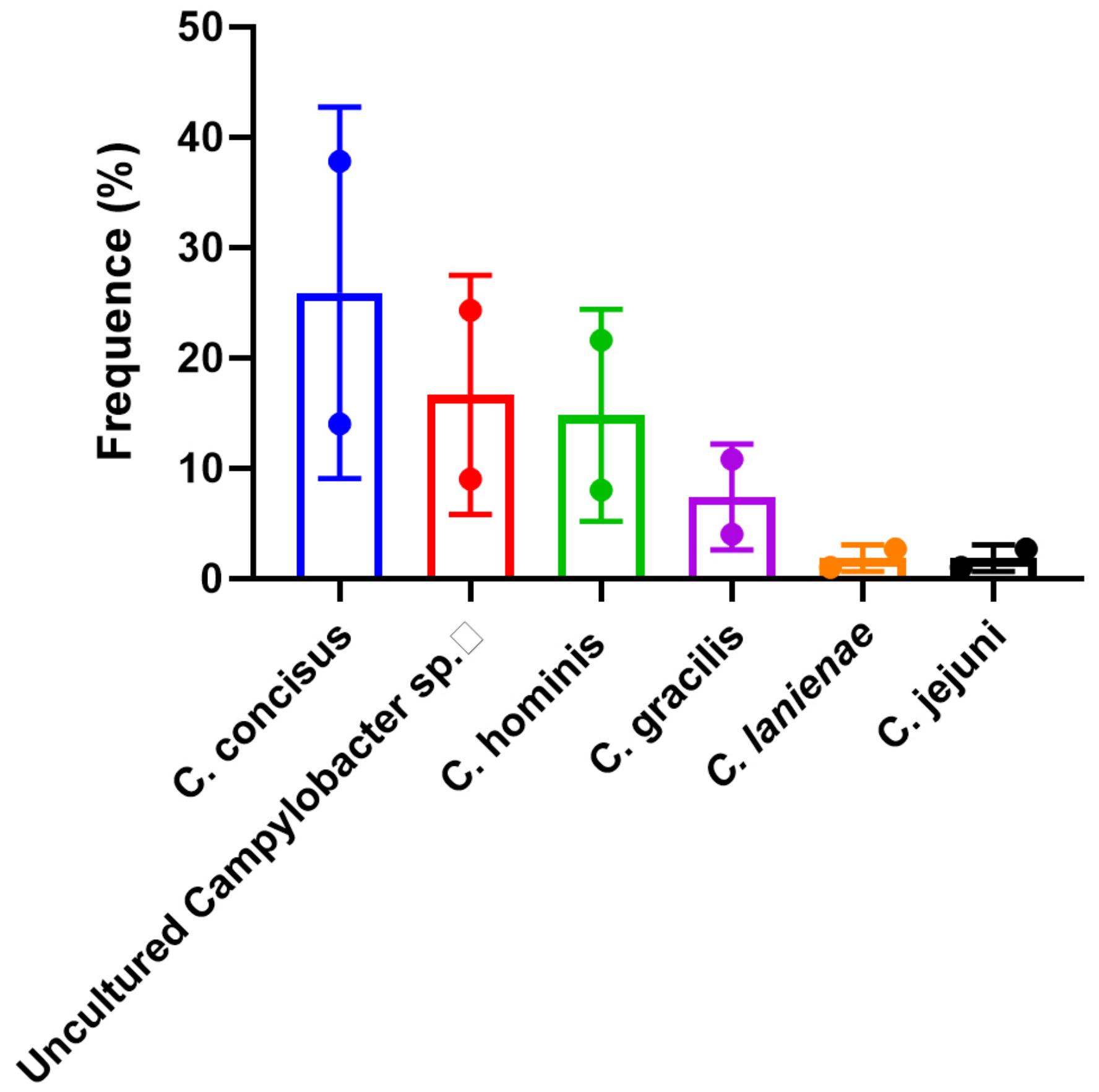

Species

Figure 2

Distribution of Campylobacter species from human samples identified by sequencing. 


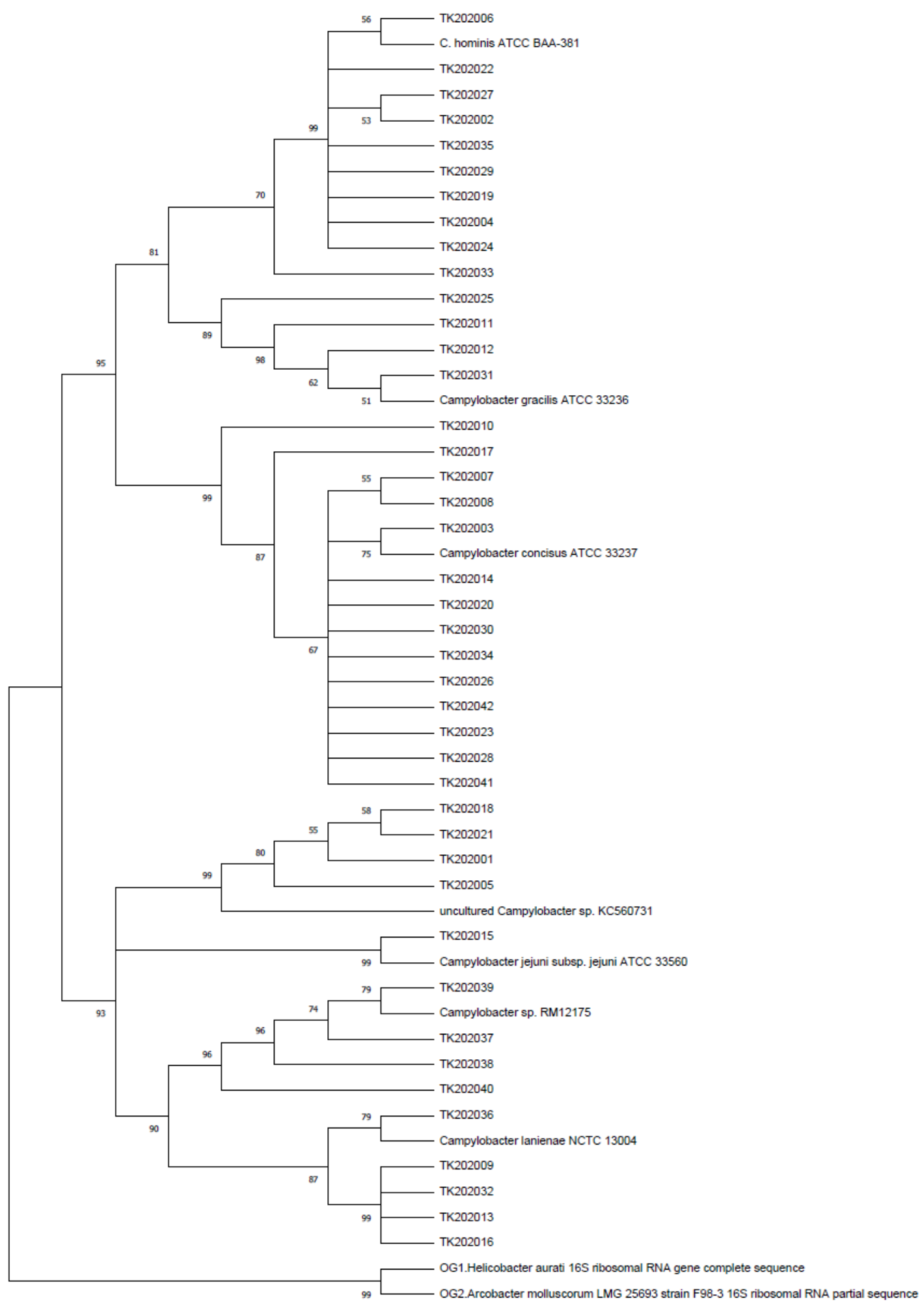

\section{Figure 3}

Molecular phylogenetic analysis of Campylobacter species using 16S rRNA sequences by the neighborjoining method. Bootstrap values (\%) based on 1000 replicates are indicated at nodes. The evolutionary distances were computed using the Jukes-Cantor method and are in the units of the number of base substitutions per site. Evolutionary analyses were conducted in MEGA X. Reference strains were included in the analysis. The tree was rooted using $\mathrm{H}$. aurati and $\mathrm{A}$. molluscorum. 